\title{
Evaluation of a rehabilitation support service after acute stroke: Feasibility and patient/carer benefit
}

\author{
Joanna J. McAdam ${ }^{1}$, Michael J. Leathley ${ }^{1}$, Margaret S. Crichton ${ }^{2}$, Julie Dickens ${ }^{3}$, \\ Cathy I. A. Jack ${ }^{4}$, Caroline L. Watkins ${ }^{1}$ \\ ${ }^{1}$ School of Health, University of Central Lancashire, Preston, UK; ${ }^{*}$ Corresponding Author: jmcadam@uclan.ac.uk \\ ${ }^{2}$ Crossroads Care (Liverpool, Sefton and Warrington), Liverpool, UK \\ ${ }^{3}$ Therapies Directorate, The Royal Liverpool and Broadgreen University Hospitals NHS Trust, Liverpool, UK \\ ${ }^{4}$ Belfast Health and Social Care Trust, Belfast, Northern Ireland
}

Received 16 April 2013; revised 17 May 2013; accepted 17 June 2013

Copyright (c) 2013 Joanna J. McAdam et al. This is an open access article distributed under the Creative Commons Attribution License, which permits unrestricted use, distribution, and reproduction in any medium, provided the original work is properly cited.

\section{ABSTRACT}

Background: Stroke survivors returning home after discharge from hospital and their carers require support to meet their rehabilitation needs (independence in Activities of Daily Living, exercise, psychosocial support). Voluntary or charitable care providers may be able to address some of these needs. Objective: To explore the feasibility of delivering and evaluating enhanced support to stroke survivors and their carers, with a Rehabilitation Support Worker (RSW). Methods: 16 consecutive stroke survivors and their carers were included. All participants received usual hospital care. Seven of these patients and their carers were also allocated an RSW from a charitable care provider. The RSW accompanied therapy training sessions with the patient, carer and therapist in hospital. On discharge, the RSW visited the patient and carer at home over the initial 6 week post-discharge period to support them in practising rehabilitation skills. Patient function (Barthel Index) and patient/carer confidence were independently assessed at discharge (Week 0). The above assessments and patient/carer mood (GHQ-12) and Carer Giver Strain were also assessed at Weeks 1, 6 and 12. RSWs were interviewed for their views about the service. Results: Participants' functional ability at Week 1 post-discharge was significantly higher in the RSW group. At 6 and 12 weeks post-discharge, functional ability was not significantly different between groups. Carers in the intervention group were less confident at all time points, however, this was not significant.
There was no significant effect on carer strain or well-being. Interviews with RSWs highlighted areas of their training that could be enhanced and the need for greater clarity as to their role. Conclusions: The results showed that a definitive trial of rehabilitation support is feasible. A number of obstacles however would need to be overcome including: difficulty in identifying suitable patients, clarity of the RSW role, and appropriate training content.

Keywords: Stroke; Rehabilitation; Activities of Daily Living; Instrumental Activities of Daily Living

\section{INTRODUCTION}

Stroke is the major cause of long-term neurological disability in adults [1] with approximately half of all those surviving living with severe functional problems [2]. The majority of stroke survivors (78\%) return to their own homes either alone or with a carer, yet of those less than $30 \%$ receive formal therapy such as seeing a Physiotherapist or Occupational Therapist [3]. Furthermore, skills developed in rehabilitation may be diminished as half of stroke survivors lose the ability to perform at least one basic functional task within six weeks of discharge [4]. Providing informal rehabilitation support upon discharge may help with these issues. In 2010, a systematic review evaluated the impact of "stroke liaison workers" and found that patients with mild to moderate disability benefited from a reduction in death and disability [5]. However, it has also been reported that studies of family or social support have failed to have much effect on functional problems [6-9]. A recent study identified that rehabilitation practice that addresses and supports autonomy, adaption and social connection may help commu- 
nity-dwelling stroke survivors regain personally valued activities post-stroke [10]. Providing a targeted rehabilitation support service designed to support promotion of activities of daily living (ADL) may therefore be more pertinent and effective.

In addition to patients' rehabilitation needs post-discharge from hospital, the needs of informal carers are also significant following a patient's return home, with carers commonly experiencing emotional distress [11] and carer strain post-discharge [12,13]. In a study of patients' and carers' rehabilitation needs, a group of five carers identified the need to provide patients with physical and mental stimuli as important [14]. In addition, the same study also identified that the needs relating to training for family members in community living were partially fulfilled [14]. Another study interviewed family care-givers of stroke survivors and suggested that implementing a family-centred model of care may better support caregivers [15]. There is therefore a clear requirement for more support for carers to address these issues in more detail. Previous studies providing family or social support have shown some benefit for carers in terms of satisfaction with practical information provision [6,9], emotional support [9], mood [6,8] and improved quality of life [6,7]. However, many have failed to improve carer mood $[9,16,17]$ or carer strain $[7,9,17]$. It is clear that possibilities exist to address the unmet needs of carers although it would appear that more could be done to improve carer strain particularly in regards to rehabilitation stimulus and resuming community living.

A rehabilitation support service provided by a locally based and well-established charitable care organisation was incorporated into a stroke service model. The service provided stroke patients and carers with a Rehabilitation Support Worker (RSW) whose role was to promote independence through providing support in ADLs and offering an opportunity for exercise. The service was intended to integrate with existing stroke care services to help address the rehabilitation needs of patients and carers post-discharge, such as practising ADLs and Instrumental Activities of Daily Living (IADL).

The aim of this trial was to test the feasibility of delivering the rehabilitation support service support service including evaluation of any beneficial effects for patients and carers, ease of recruitment and any issues relating to the role of an RSW. The trial was approved by the Liverpool Research Ethics Committee.

\section{METHODS}

Subjects were consecutive acute stroke patients who were transferred to a stroke rehabilitation unit, and their carers over an 18-month period. Patients were considered for inclusion if they were over 18 years old, discharged home to live with and be supported by a carer, had a re- sidual disability following stroke and required the assistance of one to transfer (confirmed by Multi-disciplinary Team [MDT]). The exclusion criteria for the trial were: patients discharged to residential care or home alone, patients able to independently transfer, patients unable to transfer or requiring the assistance of two, and patients with cognitive difficulties that precluded consent. The focus of the intervention was aimed at supporting the carer in practising rehabilitation skills such as ADLs (transfers, mobility skills, grooming) and also IADLs (food preparation, housework) with the patient. The inclusion/exclusion criteria therefore reflect a patient who has residual deficit with one carer supporting them.

\subsection{Procedure}

Patients who met the criteria for inclusion were identified by the stroke consultant and the ward team. For the purpose of this trial, we defined "carer" as the main person involved in every-day care of the patient other than a professional care worker. The ward manager approached the patient and carer and informed them about the trial. Written consent was obtained after 24 hours from those agreeing to participate. Consenting patient and carer units were randomised (using sealed envelopes), two weeks prior to discharge, by a third party, to either Control or Intervention group (one to one ratio).

\subsection{Control Group}

The patients in the Control group received the usual MDT, rehabilitative care, and discharge planning. Following discharge, patients received a package of conventional postdischarge support, which may have included outpatient therapy and social services output dependent on need.

\subsection{Intervention Group}

Upon consent, the patients in the Intervention group, in addition to receiving the usual MDT rehabilitative care and discharge planning outlined above, were allocated an RSW from the charitable care organisation. The organisation's usual role was to provide a carer support service, to meet the individual needs of carers and the person they are caring for. In this role, the organisation provided members of their existing care staff to be trained as RSWs. In their new role as an RSW, they received specialist stroke-relevant training with the hospital therapists, covering the medical, physical and social aspects of stroke. Training sessions included stroke awareness, the rehabilitation approach to stroke and practical aspects of stroke patient care. They were not trained to provide specific psychological support, however they were informed about these aspects in order to augment their stroke awareness. Furthermore, RSWs were not trained to practically assist in healthcare, for example hoisting the pa- 
tient or using equipment. RSWs were reminded that they were to work "with" rather than "for" the patient and carer in achieving rehabilitation goals. Written information was provided to help explain the role of an RSW.

Once trained, the RSWs attended individual, patient centred training sessions (two weeks prior to discharge) with the therapist, patient and carer, where the patient's rehabilitation goals were set. Rehabilitation activities targeted at achieving those goals were then practised by the patient in hospital, under supervision of a therapist, with observation and assistance by both the carer and the RSW. Examples of the type of activities practised include walking up and down the stairs, self-feeding and grooming (e.g. shaving). Other instrumental activities practised included food preparation (making a cup of tea) and housework (washing up). Record sheets were used and signed by therapists to ensure that RSWs had completed basic training and the patient centred training. The RSW was therefore cognisant of the patient's rehabilitation needs and was aware of the skills required by the carer to promote the patient's recovery. In order to achieve this, RSWs spent approximately six hours with the patient and carer while they were practising their rehabilitation activities.

Subsequent to discharge, the patient and carer were encouraged to continue practising the rehabilitation activities taught in hospital. They were supported in practising these activities by the RSW, who visited the patient's home on mutually agreed occasions over the first six weeks of discharge. These visits provided approximately 24 hours of rehabilitation support on a gradually reducing basis over the six week period (i.e. Weeks 1 \& 2 $=$ six hours per week, Weeks $3 \& 4=$ four hours and Weeks 5 \& 6 = two hours). RSWs were given these timings as guidelines and finished visiting the patient at the end of the 6 week period. In terms of usual care received, patients in the intervention group were not treated any differently to the control group. The trial took place before primary care based rehabilitation was routinely available to patients as part of their care package.

\subsection{Evaluation}

\subsubsection{Patient}

The focus of the intervention was aimed at supporting the patient in rehabilitation skills, primarily ADLs. Function was therefore our primary outcome, measured by the Barthel Index [18]. We were also interested in the confidence of patients in performing ADLs. We therefore measured the patient's confidence of performing each item on the Barthel (designed specifically for this trial, not psychometrically tested); each item was scored 0 (not confident) to 4 (extremely confident). Furthermore, we were interested in patient well-being: measured by the 12-item General Health Questionnaire (GHQ-12) [19].

\subsubsection{Carer}

The intervention was designed to support both patient and carer in practising rehabilitation skills. We were therefore interested in carer well-being measured by the GHQ-12 and carer strain measured by Carer Strain Index [20]. We were also interested in the level of carer confidence in the patient's ability to perform ADLs. We measured this by asking the carer to rate their confidence in the patient performing each item on the Barthel (designed specifically for this trial, not psychometrically tested), each item scored 0 (not confident) to 4 (extremely confident).

\subsubsection{Data Collection}

Data were collected face-to-face (questionnaire was read to participant) by the research physiotherapist (blind to treatment group) upon discharge (baseline) and Weeks 1,6 , and 12 post-discharge at the patient's home. Carer data were collected by leaving a questionnaire for the carer to self-complete and post back. If necessary, assistance was available to complete the carer questionnaire.

\subsubsection{RSWs}

Qualitative methods were employed to measure the RSWs' perception of the service. We asked the RSWs if they were willing to be interviewed after they had completed the intervention. Those who agreed were interviewed by a researcher on a one to one basis using a semi-structured interview guide. The interviews were designed to explore their experiences in providing the service, and areas they felt needed improvement. Interviews were transcribed verbatim and all comments were anonymised.

\subsection{Data Analysis}

Scale data were described using means and 95\% confidence intervals. Group comparisons were made using ANOVA accounting for baseline score.

Interview transcripts were read by a researcher and emergent themes were identified by coding. A second researcher independently coded the interviews and compared results to determine the reliability of themes identified.

\section{RESULTS}

Sixteen patients and carers were recruited over an 18 month period, nine (56\%) in the Control group and seven (44\%) in the Intervention group. Figure 1 shows the flow of patients through the trial and reasons for noninclusion. The collection of patients' data yielded a 100\% response. Leaving the questionnaire for the carers to complete gave us a 94\% response over the 12 weeks.

Patients in the Control group were slightly older than 


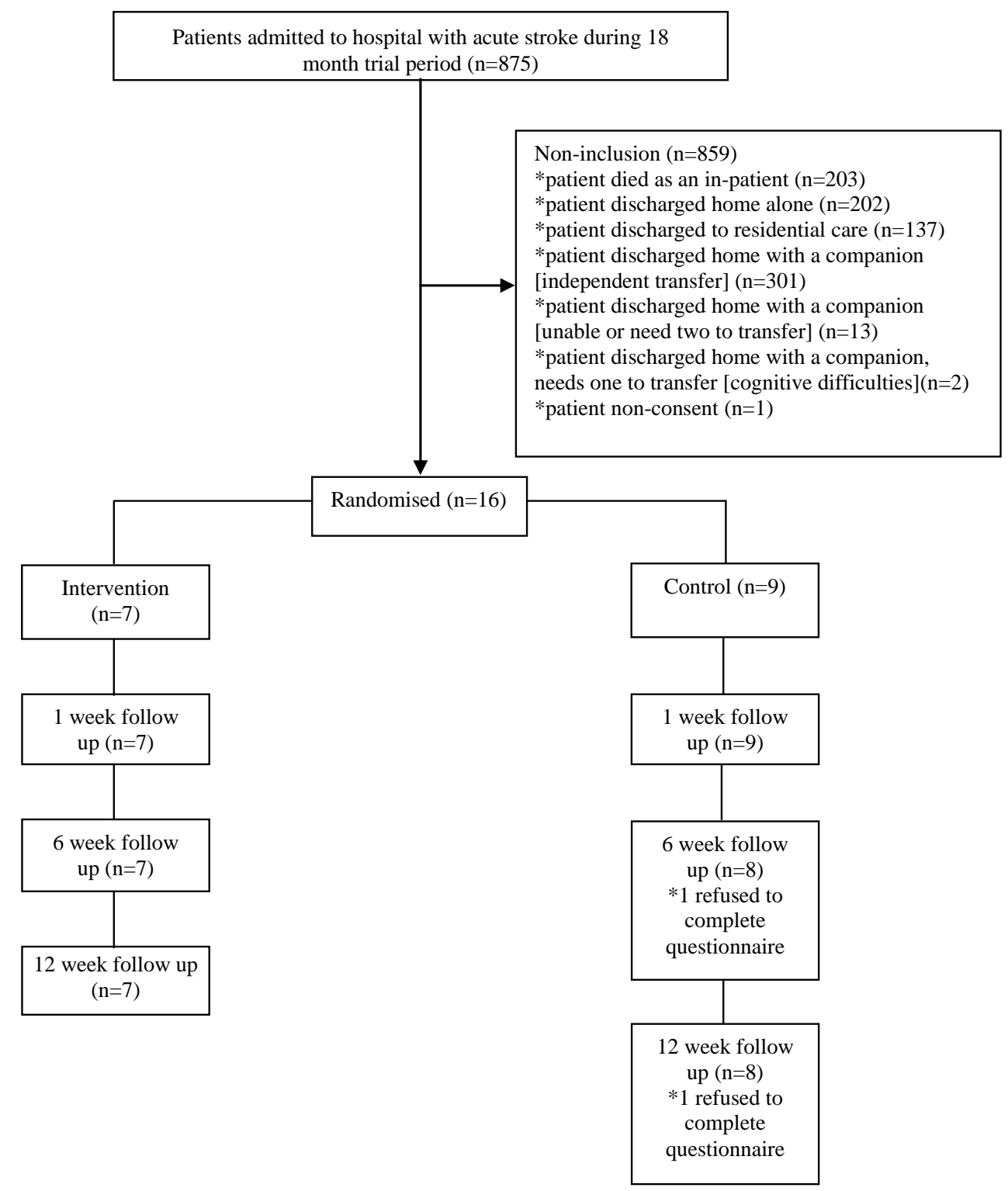

Figure 1. Flow diagram of patients entering the trial and reasons for non-inclusion.

those in the Intervention group (Table 1). There were three female patients in both the Intervention and Control group (43\% and 33\% respectively). Patients in both groups were of similar functional ability (Table 1). All patients in the trial were recruited within one to four months post-stroke. No patients died during the trial.

\subsection{Patient and Carer Outcomes}

After controlling for baseline (discharge) Barthel score, the score at week 1 (post-discharge) was significantly higher for the Intervention group (mean difference [MD $=1.6,95 \%$ confidence interval $[\mathrm{CI}]=0.5-2.7)$. At Weeks 6 and 12 there were no significant differences between the groups. There were no significant differences between groups for the Barthel score, individual
Barthel items for transfers and mobility or patient confidence in performing transfers and mobility (Table 2). Patient well-being improved between weeks 1 and 12 in the Control group and deteriorated in the Intervention group; however this was not significant (Table 2).

There were no significant differences between groups for carer well-being or carer strain (Table 3). Carers in the Intervention group were less confident than the Control group at Weeks 1, 6 and 12; however, this was not significant (Table 3).

\subsection{Interview Data}

Two RSWs agreed to be interviewed. The three main themes identified from the interviews with the RSWs were: training issues, content of the service they were 
Table 1. Baseline characteristics of patients allocated to the Intervention group (RSW) and Control group.

\begin{tabular}{lll}
\hline & Intervention group $(\mathrm{n}=7)$ & Control group $(\mathrm{n}=9)$ \\
\hline Age (years), mean & $63.7 \mathrm{SD}=14.2($ range $45-83)$ & $70.9 \mathrm{SD}=10.6$ (range $60-91)$ \\
Sex, \% male & Median 66.0 & Median 70.0 \\
Barthel Index score, mean & $4(57 \%)$ & $6(67 \%)$ \\
& $15.8 \mathrm{SD}=4.3($ range $8-20)$ & $14.9 \mathrm{SD}=3.3$ (range $9-18)$ \\
Stroke onset (days), mean & Median 16.5 & Median 16.0 \\
& $70 \mathrm{SD}=34.9($ range $26-111)$ & $52 \mathrm{SD}=14.1$ (range 30 - 82) \\
\hline
\end{tabular}

Table 2. For patients in the two groups over time, mean difference ( $95 \%$ confidence intervals) for Barthel Index total, transfers and mobility, patient confidence in transfers and mobility and patient well-being.

\begin{tabular}{|c|c|c|c|}
\hline & Week 1 & Week 6 & Week 12 \\
\hline \multicolumn{4}{|l|}{ Barthel Total } \\
\hline Usual Care & $14.4(13.7,15.1)$ & $15.6(13.2,17.9)$ & $14.7(12.1,17.2)$ \\
\hline RSW & $16.0(15.2,16.8)$ & $15.0(12.5,17.6)$ & $15.3(12.6,18.2)$ \\
\hline Difference in Means & $1.6(0.5,2.7)$ & $-0.5(-4.0,3.0)$ & $0.7(-3.1,4.5)$ \\
\hline \multicolumn{4}{|l|}{ Barthel Transfer } \\
\hline Usual Care & $2.5(2.2,2.7)$ & $2.7(2.3,3.0)$ & $2.5(2.0,3.0)$ \\
\hline RSW & $2.7(2.4,3.1)$ & $2.6(2.2,3.0)$ & $2.8(2.2,3.3)$ \\
\hline Difference in Means & $0.3(-0.2,0.7)$ & $-0.1(-0.6,0.4)$ & $0.3(-0.5,1.0)$ \\
\hline \multicolumn{4}{|l|}{ Barthel Mobility } \\
\hline Usual Care & $2.4(2.1,2.8)$ & $2.9(2.6,3.2)$ & $2.8(2.4,3.2)$ \\
\hline RSW & $2.1(1.6,2.5)$ & $2.5(2.2,2.8)$ & $2.4(2.0,2.9)$ \\
\hline Difference in Means & $-0.4(-1.0,0.2)$ & $-0.4(-0.8,0.1)$ & $-0.4(-1.0,0.3)$ \\
\hline \multicolumn{4}{|c|}{ Patient Confidence (Transfer) } \\
\hline Usual Care & $2.9(2.1,3.7)$ & $2.4(1.6,3.3)$ & $2.5(1.6,3.4)$ \\
\hline RSW & $3.5(2.6,4.5)$ & $2.8(1.9,3.7)$ & $2.4(1.4,3.5)$ \\
\hline Difference in Means & $0.6(-0.7,2.0)$ & $0.4(-0.9,1.7)$ & $-0.1(-1.5,1.4)$ \\
\hline \multicolumn{4}{|c|}{ Patient Confidence (Mobility) } \\
\hline Usual Care & $2.4(1.8,3.0)$ & $2.6(1.9,3.3)$ & $2.6(1.9,3.3)$ \\
\hline RSW & $3.2(2.3,4.2)$ & $2.4(1.5,3.3)$ & $2.6(1.7,3.4)$ \\
\hline Difference in Means & $0.9(-0.3,2.1)$ & $-0.2(-1.4,1.0)$ & $-0.1(-1.2,1.1)$ \\
\hline \multicolumn{4}{|l|}{ GHQ-12 } \\
\hline Usual Care & $5.0(2.6,7.5)$ & $3.3(1.3,5.2)$ & $3.5(0.9,6.1)$ \\
\hline RSW & $4.1(1.5,6.8)$ & $4.1(0.4,7.9)$ & $6.1(2.5,9.8)$ \\
\hline Difference in Means & $0.9(-3.1,4.8)$ & $-0.9(-5.8,4.0)$ & $-2.6(-7.7,2.4)$ \\
\hline
\end{tabular}

providing and confidence in their role.

\subsubsection{Training Issues}

It emerged that the RSWs had only had limited contact with therapists in their training period (one or two occasions) and would have been keen to have done more. They were also eager to improve their knowledge of stroke, including awareness of the differing outcomes between strokes.

\subsubsection{Content of the Service}

RSWs' perceptions of the service they were providing often conflicted with what actually happened during their visits. As a result, elements of caring for the patient were introduced to the visits.

\subsubsection{Confidence}

As a consequence of the conflicts in the content of service, it was felt that more training should be provided 
Table 3. For carers in both groups over time mean difference ( $95 \%$ confidence intervals) for confidence in patient transfers, mobility and well-being.

\begin{tabular}{|c|c|c|c|}
\hline & Week 1 & Week 6 & Week 12 \\
\hline \multicolumn{4}{|c|}{ Carer Confidence in Patient Transfer } \\
\hline Usual Care & $2.4(1.9,3.0)$ & $2.8(2.1,3.4)$ & $2.3(1.6,3.0)$ \\
\hline RSW & $2.2(1.4,3.0)$ & $2.2(1.3,3.1)$ & $1.7(0.6,2.7)$ \\
\hline Difference in Means & $-0.2(-1.2,0.8)$ & $-0.6(-1.7,0.6)$ & $-0.6(-1.9,0.6)$ \\
\hline \multicolumn{4}{|c|}{ Carer Confidence in Patient Mobility } \\
\hline Usual Care & $2.3(1.6,3.0)$ & $2.7(2.1,3.2)$ & $2.5(1.9,3.0)$ \\
\hline RSW & $2.1(1.2,2.9)$ & $2.3(1.6,2.9)$ & $1.7(0.9,2.4)$ \\
\hline Difference in Means & $-0.3(-1.4,0.9)$ & $-0.4(-1.3,0.4)$ & $-0.8(-1.7,0.2)$ \\
\hline \multicolumn{4}{|l|}{ Carer GHQ-12 } \\
\hline Usual Care & $2.3(0.6,4.0)$ & $2.6(1.0,4.2)$ & $2.5(0.9,4.1)$ \\
\hline RSW & $4.3(1.8,6.8)$ & $4.6(2.6,6.6)$ & $3.3(1.6,5.0)$ \\
\hline Difference in Means & $-2.0(-5.3,1.4)$ & $-2.0(-4.9,1.0)$ & $-0.8(-3.3,1.8)$ \\
\hline \multicolumn{4}{|l|}{ Carer Giver Strain } \\
\hline Usual Care & $3.3(1.5,5.1)$ & $5.4(2.9,7.9)$ & $5.8(2.5,9.1)$ \\
\hline RSW & $5.0(2.6,7.5)$ & $4.0(1.2,6.8)$ & $3.7(1.7,5.7)$ \\
\hline Difference in Means & $-1.7(-5.1,1.7)$ & $1.4(-2.8,5.5)$ & $2.0(-2.3,6.4)$ \\
\hline
\end{tabular}

regarding the content of each visit with the patient and carer, including awareness of different exercises and other activities. RSWs explained that this would help to improve their confidence in their role as an RSW.

\section{DISCUSSION}

The trial demonstrated a significant effect on patient function for the Intervention group at Week 1 post-discharge. However, this was not sustained at Weeks 6 or 12 and no other significant effects were reported for patient or carer outcomes. The trial showed that delivering a randomised trial of rehabilitation support post-discharge is feasible. However, there are issues in regards to recruitment to this type of rehabilitation trial, content of the intervention provided and staff training needs which need to be considered.

A significant effect was reported for the Intervention group's Barthel score at Week 1. However, the results showed no significant difference in patient's functional ability at 6 or 12 weeks post-discharge. The initial effect at Week 1 may therefore simply be reflecting the readjustment phase upon discharge that occurs to both the stroke survivor and carer. Previous studies of family support or carer training have also shown no significant effect on patient function [6-9]. The lack of effect in this trial and elsewhere suggests that rehabilitation support of this nature does not directly benefit patient function. However, this may be due to lack of a clearly defined role for the RSW. Future studies of this nature should be clear about the type of rehabilitation activities being under taken and the process of providing support.

There was no significant effect on carer confidence, carer strain or well-being. Similarly, studies of care models after stroke, such as early supported discharge or family support have also failed to improve carer mood $[9,16,17]$. The lack of effect in the carers may be a consequence of the intervention being tailored to the rehabilitation needs of the stroke survivor with no specific intervention to the carer. The intervention as such did not focus on the needs of the carer. Future studies should consider elements of the intervention designed specifically to improve carer mood and well-being.

The interview findings suggested that RSWs required specific training and development to improve their confidence in delivering the service and fulfilling their role. The RSW comments suggest that the definition of their role lacked precision. They felt that they needed more stroke-specific knowledge and a greater understanding of how to use their time with the patient and carer to greatest effect. Training plans and the content of sessions with patients and carers need to be carefully identified and planned so that the needs of RSWs, patients and carers can be met. Supervision and guidance of RSWs during the intervention period in the community may also be beneficial in making sure that RSWs are cognisant of their expectations and comfortable in their role.

Interviews also suggested that RSWs were acting in a 
caring role, rather than an enabling role. Although their role was not designed to act for the patient and carer, interviews revealed certain elements of "caring for" were introduced. A previous study of family care workers suggested that providing support rather than improving patients' coping skills may have contributed to patients in the treatment group being more helpless and possibly depressed [8]. Future studies should include more training in the enabling philosophy of stroke rehabilitation and its supporting evidence.

The trial was hampered by the small number of participants, a consequence of the difficulty in recruiting suitable patients. Our inclusion/exclusion criteria were designed to reflect the needs of a patient with a residual deficit returning home with a carer supporting them. Consequently, we excluded a large group of patients who were discharged home with a companion and who could independently transfer. Future studies of this nature should consider widening the inclusion criteria to include patients who are more mobile but have other difficulties related to ADLs or IADLs. These patients and their carers may seek benefit from an RSW service in addressing rehabilitation needs, e.g. doing housework.

\section{LIMITATIONS}

In addition to the small number of participants which limited this trial, further limitations became apparent. The choice of primary outcome measure did not take into consideration the full range of activities practised, e.g. IADLs. The rehabilitation skills practised may have been more complex than those measured by the Barthel Index (e.g. meal preparation, housework) and therefore potential benefit may not have been detected. Future work of this nature should employ additional scales to account for these methods. In addition, the nature of the community environment such as provision of other services (e.g. outpatient therapy, social input) meant that other factors may have influenced outcomes. Future trials need to identify these factors in their results. The method of collecting data face-to-face using standardized measures was found to be feasible as in a previous trial of home rehabilitation [21]. However, this method may influence patients to provide socially desirable answers [22].

\section{CONCLUSION}

Although this feasibility trial does not allow us to draw any firm conclusions as to the effectiveness of a rehabilitation support service, it has been vital for informing further evaluations with regards to the format of intervention, i.e. a more clearly defined role (including enhanced training and support) for the RSWs. It has also informed us of issues relating to the design of a trial of this nature; the number of participants suitable and the use of suitable outcome measures which accurately reflect a rehabilitation intervention.

\section{CONFLICTS OF INTEREST}

Margaret Crichton is Chair of the local Crossroads organisation that provided the Rehabilitation Support Workers.

\section{SOURCE OF FUNDING}

Royal Liverpool \& Broadgreen University Hospitals NHS Trust R\&D support fund and Crossroads Care, Liverpool, Sefton and Warrington. Liverpool Crossroads Caring for Carers Ltd is registered as a company limited by guarantee in England and Wales No 3334237. Registered charity number 1065434 CQC Reg No F020000694.

\section{REFERENCES}

[1] Wolfe, C.D. (2000) The impact of stroke. British Medical Bulletin, 56, 275-286. doi:10.1258/0007142001903120

[2] Walker, M.F., Leonardi-Bee, J., Bath, P., Langhorne, P., Dewey, M. and Corr, S. (2004) Individual patient data meta-analysis of randomized controlled trials of community occupational therapy for stroke patients. Stroke, 35, 2226-2232. doi:10.1161/01.STR.0000137766.17092.fb

[3] Watkins, C.L., Leathley, M.J. and Sharma, A.K. (2002) Stroke interface audit: Pre/post discharge audit of stroke services and care in Liverpool and Sefton: Delivery timeliness and targeting - 36 month report. University of Central Lancashire, Preston.

[4] Garraway, W.M., Akhtar, A.J., Hockey, L. and Prescott, R.J. (1980) Management of acute stroke in the elderly: Follow-up of a controlled trial. British Medical Journal, 281, 827-829. doi:10.1136/bmj.281.6244.827

[5] Ellis, G., Mant, J., Langhorne, P., Dennis, M. and Winner, S. (2010) Stroke liaison workers for stroke patients and carers: An individual patient data meta-analysis. Cochrane Database of Systematic Reviews, 5, CD005066.

[6] Kalra, L., Evans, A., Perez, I., Melbourn, A., Patel, A., Knapp, M. and Donaldson, N. (2004) Training carers of stroke patients: Randomised controlled trial. British Medical Journal, 328, 1099-1103. doi:10.1136/bmj.328.7448.1099

[7] Mant, J., Carter, J., Wade, D.T. and Winner, S. (2000) Family support for stroke: A randomised controlled trial. Lancet, 356, 808-813. doi:10.1016/S0140-6736(00)02655-6

[8] Dennis, M., O’Rourke, S., Slattery, J., Staniforth, T. and Warlow, C. (1997) Evaluation of a stroke family care worker: Results of a randomised controlled trial. British Medical Journal, 314, 1071-1076. doi:10.1136/bmj.314.7087.1071

[9] Lincoln, N.B., Francis, V.M., Lilley, S.A., Sharma, J.C. and Summerfield, M. (2003) Evaluation of a stroke fam- 
ily support organiser: A randomized controlled trial. Stroke, 34, 116-121.

doi:10.1161/01.STR.0000047850.33686.32

[10] Kubina, L.A., Dubouloz, C.J., Davis, C.G., Kessler, D. and Egan, M.Y. (2013) The process of re-engagement in personally valued activities during the two years following stroke. Disability and Rehabilitation, 35, 236-243. doi:10.3109/09638288.2012.691936

[11] Dennis, M., O’Rourke, S., Lewis, S., Sharpe, M., Warlow, C. (1998) A quantitative study of the emotional outcome of people caring for stroke survivors. Stroke, 29, 18671872. doi:10.1161/01.STR.29.9.1867

[12] Bugge, C., Alexander, H. and Hagen, S. (1999) Stroke patients' informal caregivers. Patient, caregiver and service factors that affect caregiver strain. Stroke, 30, 15171523. doi:10.1161/01.STR.30.8.1517

[13] Rouillard, S., De Weerdt, W., De Wit, L. and Jelsma, J. (2012) Functioning at 6 months post stroke following discharge from inpatient rehabilitation. South African Medical Journal, 102, 545-548.

[14] Talbot, L.R., Viscogliosi, C., Desrosiers, J., Vincent, C., Rousseau, J. and Robichaud, L. (2004) Identification of rehabilitation needs after a stroke: An exploratory study. Health and Quality of Life Outcomes, 2, 53-61. doi:10.1186/1477-7525-2-53

[15] Cameron, J.I., Naglie, G., Silver, F.L. and Gignac, M.A.M. (2013) Stroke family caregivers' support needs change across the care continuum: A qualitative study using the timing it right framework. Disability and Rehabilitation, 35, 315-324. doi:10.3109/09638288.2012.691937
[16] Langhorne, P., Taylor, G., Murray, G., Dennis, M., Anderson, C., Bautz-Holter, E., Dey, P., Indredavik, B., Mayo, N., Power, M., Rodgers, H., Ronning, O.M., Rudd, A., Suwanwela, N., Widen-Holmqvist, L. and Wolfe, C. (2005) Early supported discharge services for stroke patients: A meta-analysis of individual patients' data. Lancet, 365, 501-506.

[17] Tilling, K., Coshall, C., McKevitt, C., Daneski, K. and Wolfe, C. (2005) A family support organiser for stroke patients and their carers: A randomised controlled trial. Cerebrovascular Diseases, 20, 85-91. doi:10.1159/000086511

[18] Wade, D.T. and Collin, C. (1988) The Barthel ADL index: A standard measure of physical disability? International Disability Studies, 10, 64-67. doi:10.3109/09638288809164105

[19] Goldberg, D.P. and Hillier, V.F. (1979) A scaled version of the General Health Questionnaire. Psychological Medicine, 9, 139-145. doi:10.1017/S0033291700021644

[20] Robinson, B.C. (1983) Validation of a caregiver strain index. The Journals of Gerontology, 38, 344-348. doi:10.1093/geronj/38.3.344

[21] Anderson, C., Rubenach, S., Mhurchu, C.N., Clark, M., Spencer, C. and Winsor, A. (2000) Home or hospital for stroke rehabilitation? Results of a randomized controlled trial: 1: Health outcomes at 6 months. Stroke, 31, 10241031. doi:10.1161/01.STR.31.5.1024

[22] Duffy, B., Smith, K., Terhanian, G. and Bremer, J. (2005) Comparing data from online and face-to-face surveys. International Journal of Market Research, 47, 615-639. 